der ungefähren Himmelsrichtung, so ist das wahre Streichen von Schichten und Störungen aus dem Profil abzulesen.

Profil A gibt ein Beispiel eines solchen, auf dem eine Lagerstäte mit zwei Querstörungen dargestellt ist. Hierbei bedeutet $-90^{\circ}$, daß die Lagerstätte rechtwinklig geschnitten wird, das Vorzeichen gibt an, daß sie $90^{\circ}$ weniger von der Nord-Südlinie abweicht als die Profillinie $\left(130^{\circ}\right)$, also wirkliches Streichen $130^{\circ}-90^{\circ}=40^{\circ}$. Aus den Einschreibungen an den Störungen folgt, daß sie sehr verschieden streichen, und von dem Profil in verschieden spitzen Winkeln $20^{\circ}$ and $50^{\circ}$ geschnitten werden, also verschiedenes Fallen haben, denn je kleiner der beigefügte Winkel, um so stärker reduziert erscheint das wahre Fallen auf dem Bild. Bei einiger Übung kann man sich aus einem solchen Profil den Verlauf der Lagerstätte im Raum genau so gut vorstellen wie aus einem bergmännischen Grundriß.

\title{
Der nordalpine Flysch zwischen der Ostschweiz und Salzburg.
}

\author{
Von M. Richter (Bonn).
}

Als Ausgangspunkt dieser Besprechung dient das Allgäu-Vorarlberg. Hier sind bekanntlich zwei Flyschzonen vorhanden, eine nördliche und eine südliche, die helvetische Kreide zwischen sich lassend. Die nördliche Flyschzone besteht aus verschiedenen tektonischen Elementen, die sich auf mindestens zwei, wahrscheinlich aber auf drei helvetische Decken verteilen lassen. Damit verbindet sich diese Zone direkt mit der Fähnernmulde am Nordrand des Säntis, die ebenfalls aus mehreren tektonischen Elementen zusammengesetzt ist.

Die südliche Flyschzone ist ebenfalls helvetisch und stellt eine Mulde dar, die sich von Hindelang über Oberstdorf bis jenseits der Bregenzer Ach gut verfolgen läßt. Dadurch verbindet sie sich unmittelbar mit der AmdenWildhauser Mulde zwischen Säntis und Churfirsten. Daraus ergibt sich, daß Äquivalente der Churfirsten im Vorarlberg fehlen: die Churfirsten tauchen in ihrer östlichen Fortsetzung unter das Rhätikon unter.

Drei helvetische Decken sind im Allgäu-Vorarlberg festzustellen, von unten nach oben: Hüttenbergdecke (als Äquivalent der Mürtschendecke?), Grüntendecke und Bregenzerwalddecke, die beiden letzteren als das Äquivalent der Säntisdecke.

Die Hüttenbergdecke verschwindet nach Osten an der Iller untèr der Grüntendecke; die Bregenzerwalddecke zwischen Hindelang und Wertach unter der oberostalpinen Allgäudecke. Allein die Grüntendecke setzt nach Osten am Nordrand der bayerischen Aipen fort.

Der südbayerische und Salzburger Flysch ist ein ganz anderes Element als der Flysch der helvetischen Decken. Östlich des Lech dürfte kein helvetischer Flysch (abgesehen vom Gebiet beim Kressenberg und Mattsee) mehr vorhanden sein, da auch die Grüntendecke in der Gegend des Lech unter den südbayerischen Flysch untertaucht und nur noch in einzelnen Fenstern oder Schubfetzen an der Grenze gegen die Molasse zutage tritt.

Während der südbayerische Flysch in seiner Gesamtheit zur oberen Kreide zu stellen ist und der oberostalpinen Gosau entspricht, gehört der helvetische Flysch vom Allgäu - Vorarlberg und der Ostschweiz dem Mittel- und Obereozän, vielleicht auch noch dem untersten Oligozän an. (Mit Ausnahme des Wildflysch, der wohl der obersten Kreide zugehört.)

Südbayerischer Flysch und helvetischer Flysch stehen sich also scharf gegentiber. Der erstere wäre infolge seiner innigen Beziehungen zum Ostalpinen besser als "ostalpiner Gosauflysch" zu bezeichnen. Daß er ostalpin 
sein muß, geht aus folgendem hervor: Während der Bildung der helvetischen Oberkreide lagen die oberostalpinen Decken noch weit von der helvetischen Zone entfernt, denn oberostalpines Material fehlt ja z. B. dem Wildflysch völlig. Die oberostalpinen Decken wurden, da sie die helvetischen Falten horizontal abschneiden (z. B. im Allgäu), erst nach der Faltung der helvetischen Zone, also frühestens im Mitteloligozän, herangeschoben. (Dasselbe geht ja auch aus den Sedimenten der Molasse hervor.) Da aber der oberkretazische südbayerische Flysch infolge seiner Geröllführung an der Basis sich aufs innigste als der oberostalpinen Zone zugehörig erweist und in der Oberkreide mit der oberostalpinen Zone direkt verknüpft war, so ist er eben nicht helvetisch, sondern (ober)ostalpin. Das gleiche geht aus der tektonischen Stellung: von wahrscheinlich unterostalpinen Schubfetzen am Nordrand des südbayerischen Flysches hervor, nach denen der südbayerische Flysch höher wie unterostalpin sein muß.

Damit fällt der von manchen Anhängern der Deckenlehre postulierte prägosaujsche Schub der oberostalpinen Masse über die helvetische Zone. Diese in ihrer Gesamtheit einheitlich erfolgte Überschiebung erfolgte nicht vor dem Mitteloligozän.

Eine unmittelbare Verknüpfung der helvetischen Zone mit dem Oberostalpinen zur Gosauzeit bestand niemals, und der Einwand, der gegen die Deckenlehre wegen dieser angeblichen Verknüpfung gemacht wird, ist daher unberechtigt.

\section{Meine Hypothese einer Differentiations-Isostasie.}

\section{Von C. Mordziol (Coblenz).}

Die Grundfragen der Geologie wachsen alle aus einer einzigen, aus dem Problem der Gebirgsbildung heraus. Nun stehen aber zwei weitere Problemreihen im Hintergrunde jener Vorgänge, welche im Laufe der Erdgeschichto zur Herausbildung des primären Erdantlitzes geführt haben: das Problem des Magmaauftriebes (einerlei, ob es sich um plutonische oder vulkanische Phänomene handelt) einerseits, und die Frage nach den Ursachen der Schwereanomalien andererseits.

Zur Beantwortung diesér Fragen dürften vielleicht aussichtsreiche Forschungspläne aus einer Hypothese herauswachsen, die der Vortragende zum ersten Male in der Hauptversammlung der Geologischen Vereinigung am 9. April in Bonn kurz entwickelt ${ }^{1}$ ) hat und die als die Hypothese der Differentiations.Isostasie zu bezeichnen wäre. Die Kettengebirgsbildung wäre danach ein Teilphänomen eines magmaisostatischen Ausgleiches. Das plutonische Relief ist ein Entgasungs- und Saigerungsrelief großen Stils. Die Schwereanomalien an der Erdoberfläche sind die einfache Folge der Heransbildung jenes Saigerungsreliefs, in welchem die Ozeanbecken die Sammelpfannen schwerer basischer Magmen, die Kontinente die leichteren salischen Schlackenansammlungeu 'darstellen. Damit findet auch der Grundzug in der Abweichung des Geoids vom Referenzellipsoid seine einfache Erklärung. Der magmátische Auftrieb in den plutonischen Durchragungszonen gibt sich als hydrostatische Einstellung, einer Magma-Isostasie entsprechend, zu erkennen. Schon früh bei der Entgasung angelegte, jetzt subkrustale Saigerungsherde individualisieren in horizontalem Sinne den Differentiationsvorgang zu geognostischen Einheiten, deren Existenz schon allein aus dem geognostischen Augenschein heraus Liquation und Saigerung in der Tiefe voraussetzen läßt. Die

1) Vgl. auch: C. Mondziox, Die Gebirgsbildung der Erde. 30 S. Leipzig 1922. List \& v. Bressendorf. 